Pacific Journal of Mathematic 


\title{
EQUIVALENCE AND PERPENDICULARITY OF GAUSSIAN PROCESSES
}

\author{
JACOB FELDMAN
}

1. Introduction. In [6] S. Kakutani showed that if one has equivalent probability measures $\mu_{i}$ and $\nu_{i}$ on the $\sigma$-field $\mathscr{S}_{i}$ of subsets of a set $\Omega_{i}, i=1,2, \cdots$, and if $\mu$ and $\nu$ denote respectively the infinite product measures $\bigotimes_{i=1}^{\infty} \mu_{i}$ and $\bigotimes_{i=1}^{\infty} \nu_{i}$ on the infinite product $\sigma$-ring generated on the infinite product set $\Omega$, then $\mu$ and $\nu$ are either equivalent or perpendicular, and he obtained necessary and sufficient conditions for equivalence to occur. The theorem here shown may be regarded as a generalization of a case of the Kakutani theorem.

Similar dichotomies have revealed themselves in the study of Gaussian stochastic processes. C. Cameron and W. T. Martin proved in [2] that if one considers the measures induced on path space by a Wiener process on the unit interval, then if the variances of the processes are different the measures are perpendicular. This sort of result was generalized by U. Grenander, starting from the viewpoint of statistical estimation, and utilizing a Karhunen representation for the processes involved. A wider sufficient condition for perpendicularity of the measures induced on path space by continuous Gaussian processes on the unit interval was obtained by G. Baxter in [1]. Cameron and Martin also examined the effect on the induced measure of taking certain types of affine transformations of a Wiener process (see [3], [4]). I. E. Segal extended their results in [8], and made the situation more transparent by use of his notion of "weak distributions", and in a large class of cases got conditions for equivalence.

In the present note it is shown that the equivalence-or-perpendicularity dichotomy holds in general for pairs of measures induced by Gaussian stochastic processes, and Segal's necesssary and sufficient conditions for equivalence are extended to cover the case of nonzero mean. It has been pointed out to the author by C. Stein that one could also give a proof, in the case of zero mean, by use of the techniques of statistical testing of hypotheses.

2. Several lemmas. All Hilbert spaces mentioned will be over the reals.

Definition 1. An operator $T$ from Hilbert space $\mathbf{H}$ to Hilbert space $\mathbf{K}$ will be called on equivalence operator if

Received April 7, 1958. 
(1) $T$ is one-to-one onto, bounded, and has a bounded inverse.

(2) $\sqrt{ } T^{*} T=I+H$, where $H$ is Hilbert-Schmidt.

LEMMA 1. If $A$ is a self-adjoint bounded invertible operator on $\mathbf{H}$ then the following statements are equivalent:

(a) $A-I$ is Hilbert-Schmidt;

(b) $(A-I)^{2}$ is Hilbert-Schmidt;

( c ) $A^{-1}-I$ is Hilbert-Schmidt.

If $A, B$ satisfy (a), then so does $A B A$.

Proof. The first part is clear from consideration of the eigenvalues of the operators. For the second part: write $A=I+K, B=I+H$. Then $A B A=(I+K)^{2}+(I+K) H(I+K)$, and since the sum and product of two Hilbert-Schmidt operators is Hilbert-Schmidt, $A B A-I$ is HilbertSchmidt.

Definition 1. An operator $T$ from Hilbert space $\mathbf{H}$ to Hilbert space $\mathbf{K}$ will be called an equivalence operator if

(1) $T$ is one-to-one onto, bounded, and has a bounded inverse ;

(2) $\sqrt{ } T^{*} T-I$ is Hilbert-Schmidt.

LEMMA 2. Products, conjugates, and inverses of equivalence operators are again equivalence operators.

Proof. That they are one-to-one onto, and bounded, is clear (in the case of the conjugate operator, use the fact that the nullspace of $T^{*}$ is the orthogonal complement of the range of $T$ ).

Let $T$ be an equivalence operator from $\mathbf{H}$ to $\mathbf{K}$. Let $Q=\sqrt{T^{*} T}$. Then $V=T Q^{-1}$ is an isometry from $\mathbf{H}$ onto $\mathbf{K}$, and $T=V Q$. Thus $T^{-1}=Q^{-1} V^{*}$, and $\left(T^{-1}\right)^{*}\left(T^{-1}\right)=V Q^{-2} V^{*}$. Since $Q$ is the type of operator occurring in Lemma 1 , and $\left(T^{-1}\right)^{*} T^{-1}$ is a unitary transform of $Q^{-2}$, we get the result. Similarly, $\left(T^{*}\right)^{*} T^{*}=T T^{*}=V Q^{2} V^{*}$. Finally, let $S$ be an equivalence operator from $\mathbf{K}$ to $\mathbf{L}$, and let $P=\sqrt{S^{*} S}, U=S P^{-1}$. Then

$$
(S T)^{*}(S T)=(V Q U P)^{*}(V Q U P)=P U^{*} Q V^{*} V Q U P=P U^{*} Q U P,
$$

and again Lemma 1 tells us that $\sqrt{(S T)^{*}(S T)}$ is of the desired form.

Definition 2. A function $x$ on a measure space with measure $\mu$ of total mass 1 is called Gaussian if either or

(1) $x$ is almost everywhere a constant, $r$.

(2) there are numbers $\sigma>0$ and $\gamma$ (depending on $x$ ) such that 


$$
\mu\{\omega \mid x(\omega) \leqq \lambda\}=\frac{1}{\sqrt{ } 2 \pi \sigma} \int_{-\infty}^{\lambda} \exp \left\{\frac{1}{2}\left(\begin{array}{c}
t-\gamma \\
\sigma
\end{array}\right)^{2}\right\} d t
$$

Case 1 may be thought of as Case 2 with $\sigma=0$. Then in either case we have

$$
\int x d \mu=\gamma, \int(x-\gamma)^{2} d \mu=\sigma^{2}
$$

(the "mean" and "variance" of $x$ ).

Lemma 3. Let $\mathscr{S}_{1} \subset \mathscr{S}_{2} \subset \cdots$ be $\sigma$-fields of subsets of $\Omega$, S the smallest $\sigma$-field containing their union. Let $\mu, \nu$ be probability measures in $\mathscr{S}$ such that $\mu_{i}=\mu \mid \mathscr{S}_{i}$ is equivalent to $\nu_{i}=\nu \mid \mathscr{S}_{i}$. Let $\Lambda, \Lambda_{\mu}, \Lambda_{\nu}$ be sets in $\mathscr{S}$ forming a Hahn decomposition of $\Omega$; that is, $\mu$ is equivalent to $\nu$ when both are cut down to subsets of $\Lambda$, and $\mu\left(\Lambda_{\nu}\right)=\nu\left(\Lambda_{\mu}\right)=0$. Then $d \mu_{i} \mid d \nu_{i}$ converges almost everywhere with respect to $\mu+\nu$ to $d \mu / d \nu$, if one makes the convention $d \mu / d \nu=0$ on $A_{\nu}$ and $+\infty$ on $A_{\mu}$.

Proof. If $\Lambda_{i} \in \mathscr{S}_{i}$, then

$$
\int_{\Lambda_{i}} \frac{d \mu_{i}}{d(\mu+\nu)_{i}} d(\mu+\nu)=\mu_{i}\left(\Lambda_{i}\right)=\mu(\Lambda)
$$

so that $d \mu_{i} / d(\mu+\nu)_{i}$ is the conditional expectation of $d \mu_{i} / d(\mu+\nu)$ with respect to $\mathscr{S}_{i}$ and the measure $\mu+\nu$. Of course $\mu+\nu$ has total mass 2 , but this is inessential; one can always normalize things if so inclined. The Martingale convergence theorem then tells us that

$$
\frac{d \mu_{i}}{d(\mu+\nu)_{i}} \rightarrow \frac{d \mu}{d(\mu+\nu)}
$$

almost everywhere with respect to $\mu+\nu$. Similarly

$$
\frac{d \nu_{i}}{d(\mu+\nu)_{i}} \rightarrow \frac{d \nu}{d(\mu+\nu)}
$$

Now,

$$
\frac{d \mu_{i}}{d \nu_{i}}=\frac{d \mu_{i}}{d(\mu+\nu)_{i}} / \frac{d \nu_{i}}{d(\mu+\nu)_{i}}
$$

so

$$
\frac{d \mu_{i}}{d \nu_{i}} \rightarrow \frac{d \mu}{d(\mu+\nu)} / \frac{d \nu}{d(\mu+\nu)},
$$

where we understand the right hand side to be $+\infty$ when the 
denominator but not the numerator is zero. Now $d \mu / d(\mu+\nu)$ vanishes precisely on $\Lambda \mu$, and $d \nu / d(\mu+\nu)$ vanishes precisely on $A_{\mu}$, all statements being up to $(\mu+\nu)$ - measure 0 . Whence the lemma.

The following fact is known, and we list it for reference :

Lemma 4. If $z_{i}, z_{2}, \cdots$ are measurable functions with independent Gaussian distributions, mean 0, variance 1, then the product

$$
a_{1} \cdots a_{k} \exp \left\{\frac{1}{2} \sum_{i=1}^{n}\left(1-a_{i}^{2}\right) Z_{i}^{2}\right\}
$$

converges to zero almost everywhere if

$$
\sum_{i=1}^{\infty}\left|1-\alpha_{i}^{2}\right|^{2}=+\infty,
$$

and converges to a finite non-zero limit almost everywhere if

$$
\sum_{i=1}^{\infty}\left|1-a_{i}^{2}\right|^{2}<\infty
$$

This can be proven, for example, by applying Kakutani's conditions in [6] for equivalence of product measures.

Lemma 5. Let $R$ be a closed densely defined linear operator from the Hilbert space $\mathbf{H}$ to the Hilbert space $\mathbf{K}$. Then there is an equivalence operator $U$ from $\mathbf{H}$ onto $\mathbf{H}$ such that $U^{*} R^{*} R U$ has pure point spectrum.

Proof. Let $\sqrt{R^{*} R}=\int_{0}^{\infty} \lambda d F(\lambda)$. Let $F_{i}=F\left(2^{i}\right)-F\left(2^{i-1}\right), \quad i=$ $0, \pm 1, \pm 2 \cdots$, and let $R_{i}=\sqrt{R^{*} R} \mid \mathbf{H}_{i}$, where $\mathbf{H}_{i}$ is the range of $F_{i}$. By a theorem of von Neumann in [7] there is a self-adjoint HilbertSchmidt operator $H_{i}$ in $\mathbf{H}_{i}$ whose Hilbert-Schmidt norm $\left\|H_{i}\right\|_{2} \leqq 2^{-2|i|-3}$, and such that $R_{i}+H_{i}$ has pure point spectrum. Now consider the equation $R_{i}\left(I+K_{i}\right)=R_{i}+H_{i}$, that is $R_{i} K_{i}=H_{i}$. Since $R_{i}$ is invertible in $\mathbf{H}_{i}$, and, in fact, $\left\|R_{i}\right\|^{-1} \leqq 2^{-i+1}$, we get a solution $K_{i}=R_{i}^{-1} H_{i}$, and

$$
\left\|K_{i}\right\|_{2} \leqq\left\|R_{i}^{-1}\right\|\left\|H_{i}\right\|_{2} \leqq 2^{-i+1} \cdot 2^{-2|i|-3} \leqq 2^{-|i|-2} .
$$

Let $K$ be defined on $\mathbf{H}$ by setting $K \mid \mathbf{H}_{i}=K_{i}$. Then

$$
\|K\|_{2}^{2}=\sum_{-\infty<i<+\infty}\left\|K_{i}\right\|_{2}^{2} \leqq \sum_{-\infty<i<+\infty} 2^{-|i|-2}=3 / 4 .
$$

so $K$ is Hilbert-Schmidt, and $U=I+K$ is an equivalence. Further, $\left(I+K_{i}\right)^{*} R_{i}^{2}\left(I+K_{i}\right)$ has a complete set of eigenvectors in $\mathbf{H}_{i}$. But this operator is precisely the restriction of $U^{*} R^{*} R U$ to $\mathbf{H}_{i}$. Therefore $U^{*} R^{*} R U$ has a complete set of eigenvectors in $\mathbf{H}$. 
We shall be considering linear spaces of Gaussian functions. In taking the closures of such linear spaces in the $\mathbf{L}_{2}(\mu)$ norm, the functions obtained as limits will again be Gaussian, as is well known and easy to show, the means and variances of a limit being in fact limits of the means and variances of the approximating Gaussian functions. Furthermore, the topology of convergence in measure on Gaussian functions agrees with $\mathbf{L}_{2}(\mu)$-topology. This is shown in the mean zero case in [8], and the general case can be reduced to this by showing the following:

Lemma 6. Let $x_{i}$ be a net of $\mu$-measurable functions with Gaussian distributions, converging in probability to zero. Then their means $\gamma_{i}$ converge to zero.

Proof. Suppose this does not occur. Then by cutting down to a subnet if necessary, and occasionally using $-x_{i}$ instead of $x_{i}$ if necessary, we can assume that there is some $c>0$ such that $\gamma_{i} \geqq c$ for all $i$. Now,

$$
\mu\left\{\omega \mid x_{i}(\omega)-\gamma_{i}>0\right\}=\mu\left\{\omega \mid x_{i}(\omega)-\gamma_{i}<0\right\}
$$

so that

$$
\begin{aligned}
\mu\left\{\omega \mid x_{i}(\omega)>c\right\} & \geqq \mu\left\{\omega \mid x,(\omega)>\gamma_{i}\right\}=\mu\left\{\omega \mid x_{i}(\omega)<\gamma_{i}\right\} \\
& \geqq \mu\left\{\omega \mid x_{i}(\omega)<c\right\} \geqq \mu\left\{\omega|| x_{i}(\omega) \mid<c\right\} .
\end{aligned}
$$

The sets on the two ends of the inequality are disjoint, and that on the small end has measure converging to 1 , which gives the desired contradiction.

Lemma 7. Let $\mu, \nu$ be nonperpendicular measures. Suppose $x_{i}$ is Gaussian with respect to $\mu$ and $\nu, x_{i} \rightarrow 0$ in $\mu$-measure, and $x_{i} \rightarrow x$ in

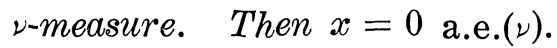

Proof. Since $x$ is Gaussian under $\nu$, the assumption that it is not zero a.e.( $\nu$ implies that it is invertible a.e.( $\nu)$. Then $x_{i} x^{-1} \rightarrow 1$ in $\nu$ measure, whereas $x_{\dot{z}} \rightarrow 0$ a.e. $(\mu)$, which implies $\mu \perp \nu$.

\section{The theorem.}

THEOREM Let $\mathbf{L}$ be a linear space of real-valued functions on a set $\Omega$. Let $\mathscr{S}$ be the smallest $\sigma$-field of subsets of $\Omega$ with respect to which all the functions in $\mathbf{L}$ are measurable. Let $\mu$ and $\nu$ be probability measures on $\mathscr{S}$. Suppose all the functions of $\mathbf{L}$ are Gaussian via both measures. Then either $\mu \sim \nu$ or $\mu \perp \nu$. Necessary and sufficient for equivalence is that if we let $\mathbf{K}$ be the linear space generated by $\mathbf{L}$ and the real-valued constant functions, then the $\mu$-equivalence clusses of functions in $\mathbf{K}$ are 
the same as the v-equivalence classes, and the identity correspondence between the two types of equivalence classes in $\mathbf{K}$ is induced by an equivalence operator between the $\mathbf{L}_{2}(\mu)$-closvre of the $\mu$-equivalence classes and the $\mathbf{L}_{2}(\nu)$-closure of the v-equivalence classes.

Proof. First, assume $\mu$ not $\perp \nu$. Let $\mathbf{J}=\left\{x-\int x d \mu \mid x \varepsilon \mathbf{L}\right\}$. For any function $x$, let $x^{\mu}$ (respectively $x^{\nu}$ ) denote the equivalence class of $x$ modulo functions which are $\mu$-null (respectively $\nu$-null), and, for a set $\mathbf{S}$ of functions, let $\mathbf{S}_{\nu}, \mathbf{S}_{\mu}$ denote the corresponding set of equivalence classes. $\quad \overline{\mathbf{S}}_{\mu}$ will mean the $\mathbf{L}_{2}(\mu)$ closure of $\mathbf{S}$.

All elements in $\mathbf{K}$ are Gaussian under $\mu$ and $\nu$, and the correspondence $x^{\mu} \leftrightarrow x^{\nu}$ between $\mathbf{K}_{\mu}$ and $\mathbf{K}_{\nu}$ is one-to-one and closable, by Lemma 5. So there is a one-to-one closed operator $T$ from a dense subspace $\mathbf{D}_{T}$ of $\overline{\mathbf{K}}_{\mu}$ to a dense subspace $\mathbf{R}_{T}$ of $\overline{\mathbf{K}}_{\nu}$ such that $T x^{\mu}=x^{\nu}$ for all $x$ in K. Further, given any $\xi$ in $\mathbf{D}_{T}$, there is some $\mathscr{S}$-measurable $x$ such that $\xi=x^{\mu}$ and $T \xi=x^{\nu}$. For choose $x_{i}$ in $\mathbf{K}$ such that $x_{i}^{\mu} \rightarrow \xi, x_{i}^{\nu} \rightarrow T \xi$. By taking subsequences, the convergence can be made a.e. $(\mu)$ and a.e. $(\nu)$ respectively, so that $\xi$ and $T \xi$ must agree a.e. $(\mu \wedge \nu)$.

Let $S=T \mid \mathbf{D}_{T} \cap \bar{J}_{\mu}$. Then $S$ is closed, with dense domain in $\bar{J}_{\mu}$ and dense range in $\bar{J}_{\nu}$, by Lemma 6 . Lemma 5 gives us an equivalence $U$ in $\bar{J}_{\mu}$ such that $U^{*} S^{*} S U$ has pure point spectrum. Choose $y_{1}, y_{2}, \cdots$ such that the $y_{i}^{\mu}$ are a complete orthonormal set of eigenvectors for $U^{-1 *} U^{-1}$, with eigenvalues $a_{i}^{2}$. Then the vectors $U^{-1} y_{i}^{u}$ are again orthogonal, and $\left\|U^{-1} y_{i}^{\mu}\right\|=a_{i}^{-1}$. Let $\mathscr{S}_{N}$ be the sample space of $y_{1}, \cdots, y_{N}$. Put a new measure $\mu^{\prime}$ on $\mathscr{S}$ by letting $y_{1}, y_{2}, \cdots$ be Gaussian, independent, mean 0 , variance $1 / a_{1}^{2}, 1 / a_{2}^{2}, \cdots$ Then

$$
\frac{d \mu^{\prime} \mid \mathscr{S}_{N}}{d \mu \mid \mathscr{S}_{N}}=a_{1} \cdots a_{N} \exp \left\{\frac{1}{2} \sum_{i=1}^{N}\left(1-a_{i}^{2}\right) y_{i}^{2}\right\},
$$

which converges almost everywhere $(\mu+\nu)$ to a nonzero limit, so that $\mu^{\prime} \sim \mu$.

Now we wish to show $\mu^{\prime} \sim \nu$. We have $x^{\nu}=S x^{\mu}$ for $x \in \mathbf{J}$, so $x^{\nu}=$ $(S U) U^{-1} x^{\mu}=(S U) x^{\mu^{\prime}}$. Let $S^{\prime}=S U$. Then, taking a.e. limits on both sides, one can for every $\xi$ in $\mathbf{D}_{S^{\prime}}$ find some $\mathscr{S}$-measurable $x$ such that $x^{\mu^{\prime}}=\xi$ and $x^{\nu}=S^{\prime} \xi$. Now choose functions $Z_{1}, Z_{2}, \cdots$ such that $Z_{i}^{\mu^{\prime}}$ form a complete orthonormal set of eigenvalues for $S^{\prime *} S^{\prime}$. The $S^{\prime} Z_{i}^{\mu^{\prime}}=Z_{i}^{\nu}$ are also orthogonal and $\operatorname{span} \mathbf{J}_{\nu}$. Define $\int Z_{i} d_{\nu}=\gamma_{i}$ and $\int\left|Z_{i}\right|^{2} d \nu^{\prime}=\alpha_{i}^{2}$. Then $\alpha_{i}$ is never zero, since $S^{\prime}$ is nonsingular, and $\int\left(Z_{i}-\gamma_{i}\right)\left(Z_{j}-\gamma_{j}\right) d \nu=$ $\alpha_{i}^{2} \delta_{i j}-\gamma_{i} \gamma_{j}$, where $\delta_{i j}$ is the Kronecker delta function. So the covariance matrix of $Z_{1}, \cdots Z_{N}$ in $\nu$-measure is given by $C_{N}=A_{N}^{2}-\vec{\gamma}_{N} \otimes \vec{\gamma}_{N}$, where $A_{N}$ is the matrix 


$$
\left(\begin{array}{ll}
\alpha_{1} & \\
& \ddots \\
& \alpha_{N}
\end{array}\right)
$$

$\vec{\gamma}_{N}$ is the vector $\left(\gamma_{1}, \cdots, \gamma_{N}\right)$, and the notation $\vec{\gamma}_{N} \otimes \vec{\gamma}_{N}$ represents a dyadic operator. $C_{N}$ is, of course, nonnegative definite. Let $\delta_{i}=\gamma_{i} / \alpha_{i}$, and $\vec{\delta}_{N}=\left(\delta_{1}, \cdots, \delta_{N}\right)$ So $\vec{\delta}_{N}=A_{N}^{-1} \vec{\gamma}_{N}$, and $A_{N}^{-1} C_{N} A_{N}^{-1}=I-\vec{\delta}_{N} \otimes \vec{\delta}_{N}$ is a nonnegative definite matrix. By conjugating with an orthogonal matrix, this can be transformed into the equivalent matrix $I-\left\|d_{N}\right\|^{2} E$, where

$$
E=\left(\begin{array}{cccc}
1 & 0 & \cdots & 0 \\
0 & 0 & \cdots & 0 \\
0 & \cdots & 0 & 0
\end{array}\right)
$$

The determinant of this, and hence of $I-\vec{\delta}_{N} \otimes \vec{\delta}_{N}$, is $I-\left\|\vec{\delta}_{N}\right\|^{2}$. Thus

$$
\left|C_{N}\right|=\left|A_{N}\right|^{2}\left|1-\left\|\vec{\delta}_{N}\right\|^{2}\right|=\alpha_{1}^{2} \cdots \alpha_{N}^{2}\left(1-\sum_{i=1}^{N} \delta_{i}^{2}\right) .
$$

Observe that

$$
\delta_{i}=\frac{\gamma_{i}}{\alpha_{i}}=\frac{\left(Z_{i}, 1\right)}{\sqrt{\left(Z_{i}, Z_{i}\right)}},
$$

where $(\cdot, \cdot)$ denotes the inner product in $\mathbf{L}_{2}(\nu)$, so that, since $1 \notin \overline{J_{\nu}}, \sum \delta^{2}<(1,1)=1$. Thus $\left|C_{N}\right| \neq 0$, and $C_{N}$ is nonsingular. The inverse matrix to $C_{N}$ is

$$
C_{N}^{-1}=\left(A_{N}^{*}-\vec{\gamma}_{N} \otimes \vec{\gamma}_{N}\right)^{-1}=A_{N}^{-2}+\frac{A_{N}^{-2} \vec{\gamma}_{N} \otimes A_{N}^{-2} \vec{\gamma}_{N}}{1-\left\|A_{N}^{-1} \vec{\gamma}_{N}\right\|^{2}} .
$$

Now let $\mathscr{J}_{N}$ be the sample space of $Z_{1}, \cdots, Z_{N}$ and let $\mu_{N}=\mu^{\prime} \mid \mathscr{J}_{N}$, $\nu_{N}=\nu \mid \mathscr{J}_{N}$. Then $d \nu_{N} / d \mu_{N}$ is precisely

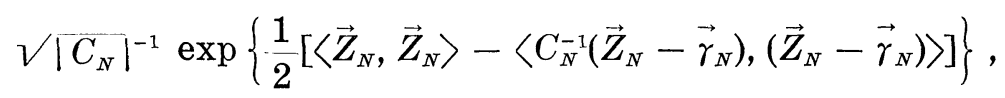

where $\vec{Z}_{N}=\left(Z_{1}, \cdots, Z_{N}\right)$. A calculation shows that the exponent can be written

$$
\frac{1}{2}\left[\left\|\vec{Z}_{N}\right\|^{2}-\left\|A_{N}^{-1} \vec{Z}_{N}\right\|^{2}-\frac{1}{1-\left\|\vec{\delta}_{N}\right\|^{2}}\left(\left\langle\vec{\delta}_{N}, A_{N}^{-1} \vec{Z}_{N}\right\rangle-1\right)^{2}-1\right] .
$$

Consider the convergence of $d \nu_{N} / d \mu_{N}$. At this stage one could already conclude from the zero-one law that $\left\{\omega \mid d \nu_{N} / d \mu_{N}(\omega) \rightarrow 0\right\}$ has measure 0 or 1 , since the set is independent of $Z_{1}, \cdots, Z_{N}$ for each $N$. However, we wish to get precise conditions for when this occurs. 
(a) Suppose

$$
\sum_{i=1}^{\infty}\left|\frac{1}{\alpha_{i}^{2}}-1\right|^{2}=\infty
$$

Consider the factor

$$
\begin{aligned}
& V\left|\bar{C}_{N}\right|^{-1} \exp \left\{\frac{1}{2}\left[\left\|\vec{Z}_{N}\right\|^{2}-\left\|A_{N}^{-1} \vec{Z}_{N}\right\|^{2}\right\}\right. \\
& =\left(1-\sum_{i=1}^{N} \delta_{i}^{2}\right)^{-1 / 2}\left(\frac{1}{\alpha_{i}} \cdots \frac{1}{\alpha_{N}}\right) \exp \frac{1}{2} \sum_{i=1}^{N}\left(1-\frac{1}{\alpha_{i}^{2}}\right) Z_{i}^{2} .
\end{aligned}
$$

Applying Lemma 3, this factor converges to zero almost everywhere with respect to $\mu^{\prime}$. The other factor of $d \nu_{N} / d \mu_{N}$, namely

$$
\exp \frac{1}{2}\left\{-\left(\frac{\left\langle\vec{\delta}_{N}, A_{N}^{-1} \vec{Z}_{N}\right\rangle-1}{1-\left\|\delta_{N}\right\|^{2}}\right)^{2}-1\right\}
$$

is clearly bounded above, so in this case $d \nu_{N} / d \mu_{N} \rightarrow 0$.

(b) Suppose

$$
\sum_{i=1}^{\infty}\left|\frac{1}{\alpha_{i}^{2}}-1\right|^{2}<\infty .
$$

Then, again by Lemma 3 , the factor

$$
\frac{1}{\alpha_{1} \cdots \alpha_{N}} \exp \frac{1}{2} \sum_{i=1}^{N}\left(1-\frac{1}{\alpha^{2}}\right) Z_{i}^{2}
$$

converges almost everywhere $\mu^{\prime}$ to a finite limit. The remaining factor is, except for a constant,

$$
\frac{1}{1-\left\|\vec{\delta}_{N}\right\|^{2}}=\exp \frac{1}{2}\left\{-\frac{1}{1-\left\|\delta_{N}\right\|^{2}}\left(\sum_{i=1}^{N} \frac{\delta_{i} Z_{i}}{\alpha_{i}}-1\right)^{2}\right\}
$$

Since $\sum_{i=1}^{\infty} \delta_{i}^{2}<1$, everything in sight converges, because $\sum_{i=1}^{\infty}\left(\delta_{i} / \alpha_{i}\right)^{2}<\infty$. So $\mu^{\prime} \sim \nu$, and $S^{\prime}$ is an equivalence from $\overline{\mathbf{J}}_{\mu}$ to $\overline{\mathbf{J}}_{\%}$. Then $S$ is likewise, and therefore $T$ is an equivalence operator.

Conversely if $\mathbf{L}$ consists of Gaussian functions under $\mu$ and $\nu$, and the correspondence $x_{\mu}+c \leftrightarrow x_{\nu}+c, x \in \mathbf{L}$, is the restriction of an equivalence operator $T$ from $\mathbf{K}_{\mu}$ to $\mathbf{K}_{\nu}$, then again choosing a basis of eigenvectors for $T \mid \overline{\mathbf{J}}_{\mu}$, we get convergence of the Radon-Nikodym derivatives to a non-zero limit, because of Lemma 3, and therefore get equivalence of the induced measures.

4. An example. Let $T$ be a set, $\Omega$ the set of all real-valued functions 
on $T$. Let $x_{t}(\omega)=\omega(t)$, and let $\mathscr{S}$ be the smallest $\sigma$-algebra with respect to which all the $x_{t}$ are measurable. Let $\mu, \nu$ be measures on $\mathscr{S}$, by each of which $x_{t}$ becomes a Gaussian stochastic process. Let

$$
\begin{gathered}
m(t)=\int x_{t} d \mu, \rho(s, t)=\int x_{s} x_{t} d \mu-m(s) m(t), \\
n(t)=\int x_{t} d_{\nu}, \sigma(s, t)=\int x_{s} x_{t} d \nu-n(s) n(t) .
\end{gathered}
$$

Let $\tau$ be a measure on $T$ such that $\rho, \sigma, m, n$, become measurable. Define, for $\tau$-measurable $f$

$$
\begin{aligned}
& {[f, f]_{\mu}=\iint(\rho(s, t)+m(s) m(t)) f(s) f(t) d \tau(s) d \tau(t),} \\
& {[f, f]_{\nu}=\iint(\sigma(s, t)+n(s) n(t)) f(s) f(t) d \tau(s) d \tau(t),}
\end{aligned}
$$

and $[f, f]=[f, f]_{\mu}+[f, f]_{\nu}$. Let $\mathbf{L}_{0}$ be those $f$ for which $[f, f]<\infty$. Then we get inner products $[f, g]_{\mu}$, etc. on $\mathbf{L}_{0}$. Define $\int f(t) x_{t} d \tau(t)$ as an $\mathbf{L}_{2}(\mu+\nu)$ valued integral, for $f \in \mathbf{L}_{0}$. This can be done, and in fact

$$
\begin{aligned}
& {[f, g]_{\mu}=\int\left(\int f(t) x_{t} d \tau(t)\right)\left(\int g(t) x_{s} d \tau(s)\right) d \mu,} \\
& {[f, g]_{\nu}=\int\left(\int f(t) x_{t} d \tau(t)\right)\left(\int g(s) x_{s} d \tau(s)\right) d \nu .}
\end{aligned}
$$

Let $\mathbf{L}=\left\{x \mid\right.$ there is some $f$ in $\mathbf{L}_{0}$ for which $x$ has as its $\mu+\nu$ equivalence class $\left.\int f(t) x_{t} d \tau(t)\right\}$. $\mathbf{L}$ is a linear set of functions, all Gaussian with respect to either $\mu$ or $\nu$. Let $\mu_{0}=\mu \mid \mathscr{S}_{0}$ and $\nu_{0}=\nu \mid \mathscr{S}_{0}$. We know from our theorem that $\mu_{0}$ and $\nu_{0}$ are either equivalent or perpendicular. Let $\mathbf{H}$ and $\mathbf{K}$ be the Hilbert spaces gotten by completing the $\mu$-equivalence classes of $\mathbf{L}_{0}$ in $\mathbf{L}_{2}(\mu)$ and the $\nu$-equivalence classes in $\mathbf{L}_{2}(\nu)$. The inner products then come from $[f, g]_{\mu}$ and $[f, g]_{\nu}$. Necessary for equivalence is that the identity map on $\mathbf{L}_{0}$ induce an equivalence operator from $\mathbf{H}$ to $\mathbf{K}$, and in order to get sufficiency we just have to be sure that if $\mathbf{L}_{0}$ is enlarged to include multiples of the identity, the identity map still induces an equivalence operator on the Hilbert spaces. This amounts to requiring that 1 be an $\mathbf{L}_{2}(\mu)$ limit of functions in $\mathbf{L}_{3}$ if and only if it is also an $\mathbf{L}_{2}(\nu)$ limit.

\section{BIBLIOGRAPHY}

1. Baxter : A sirong limit theorem for Gaussian processes, Proc. Amer. Math. Soc., (1956).

2. Cameron and Martin: The behavior of measurabilty under change of scale Wiener space, Bull. Amer. Math., Soc., (1947).

3. Cameron and Martin: Transformaition of Wiener integrals under translation of Wiener integrals under translation, Ann. Math., Stat. (1944). 
4. Cameron and Martin: Transformation of Wiener integrals under a general class of linear transformations, Trans. Amer. Math. Soc., 1945.

5. Grenander: Stochastic processes and statistical inference, Arkiv für Matematik, Band 1, nr. 17.

6. S. Kakutani: On equivalence of infinite product measures, Ann. Math. Stat., 1948.

7. J. von Neumann: Charakterisierung des Spekirums eines Integral-operatoren, Actualités Sci. Ind. 229, Paris (1935).

8. L. E. Segal: Distributions in Hilbert spaces and canonical systems of operators (from privately distributed mimeographed notes).

UNIVERSITY OF CALIFORNIA, BERKELEY 


\section{PACIFIC JOURNAL OF MATHEMATICS}

\section{EDITORS}

\section{David Gilbarg}

Stanford University

Stanford, California

\section{R. A. Beaumont}

University of Washington

Seattle 5, Washington

\author{
A. L. Whiteman
}

University of Southern California Los Angeles 7, California

E. G. Straus

University of California

Los Angeles 24, California

\section{ASSOCIATE EDITORS}

\author{
E. F. BECKENBACH \\ C. E. BURGESS \\ M. HALL \\ E. HEWITT
}
A. HORN
V. GANAPATHY IYER
R. D. JAMES
M. S. KNEBELMAN
L. NACHBIN
I. NIVEN
T. G. OSTROM
H. L. ROYDEN

M. M. SCHIFFER

G. SZEKERES

F. WOLF

K. YOSIDA

\section{SUPPORTING INSTITUTIONS}

\author{
UNIVERSITY OF BRITISH COLUMBIA \\ CALIFORNIA INSTITUTE OF TECHNOLOGY \\ UNIVERSITY OF CALIFORNIA \\ MONTANA STATE UNIVERSITY \\ UNIVERSITY OF NEVADA \\ OREGON STATE COLLEGE \\ UNIVERSITY OF OREGON \\ OSAKA UNIVERSITY \\ UNIVERSITY OF SOUTHERN CALIFORNIA
}

\author{
STANFORD UNIVERSITY \\ UNIVERSITY OF TOKYO \\ UNIVERSITY OF UTAH \\ WASHINGTON STATE COLLEGE \\ UNIVERSITY OF WASHINGTON \\ * * * * \\ AMERICAN MATHEMATICAL SOCIETY \\ CALIFORNIA RESEARCH CORPORATION \\ HUGHES AIRCRAFT COMPANY \\ THE RAMO-WOOLDRIDGE CORPORATION
}

Mathematical papers intended for publication in the Pacific Journal of Mathematics should be typewritten (double spaced), and the author should keep a complete copy. Manuscripts may be sent to any one of the four editors. All other communications to the editors should be addressed to the managing editor, E. G. Straus at the University of California, Los Angeles 24, California.

50 reprints per author of each article are furnished free of charge; additional copies may be obtained at cost in multiples of 50 .

The Pacific Journal of Mathematics is published quarterly, in March, June, September, and December. The price per volume (4 numbers) is $\$ 12.00$; single issues, $\$ 3.50$. Back numbers are available. Special price to individual faculty members of supporting institutions and to individual members of the American Mathematical Society: $\$ 4.00$ per volume; single issues, $\$ 1.25$.

Subscriptions, orders for back numbers, and changes of address should be sent to Pacific Journal of Mathematics, 2120 Oxford Street, Berkeley 4, California.

Printed at Kokusai Bunken Insatsusha (International Academic Printing Co., Ltd.), No. 6, 2-chome, Fujimi-cho, Chiyoda-ku, Tokyo, Japan.

PUBLISHED BY PACIFIC JOURNAL OF MATHEMATICS, A NON-PROFIT CORPORATION

The Supporting Institutions listed above contribute to the cost of publication of this Journal, but they are not owners or publishers and have no responsibility for its content or policies. 


\section{Pacific Journal of Mathematics}

\section{Vol. 8, No. $4 \quad$ June, 1958}

Richard Arens, The maximal ideals of certain functions algebras ........ 641

Glen Earl Baxter, An operator identity ........................... 649

Robert James Blattner, Automorphic group representations ........... 665

Steve Jerome Bryant, Isomorphism order for Abelian groups ............ 679

Charles W. Curtis, Modules whose annihilators are direct summands...... 685

Wilbur Eugene Deskins, On the radical of a group algebra ............ 693

Jacob Feldman, Equivalence and perpendicularity of Gaussian

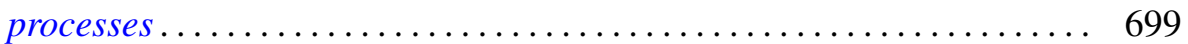

Marion K. Fort, Jr. and G. A. Hedlund, Minimal coverings of pairs by triples....................................... 709

I. S. Gál, On the theory of $(m, n)$-compact topological spaces ......... 721

David Gale and Oliver Gross, A note on polynomial and separable games........................................ 735

Frank Harary, On the number of bi-colored graphs ............... 743

Bruno Harris, Centralizers in Jordan algebras ................... 757

Martin Jurchescu, Modulus of a boundary component ............... 791

Hewitt Kenyon and A. P. Morse, Runs . . . . . . . . . . . . . . . . . . . . . . 811

Burnett C. Meyer and H. D. Sprinkle, Two nonseparable complete metric

spaces defined on $[0,1] \ldots \ldots \ldots \ldots \ldots \ldots \ldots \ldots \ldots \ldots \ldots . \ldots . \ldots . \ldots . \ldots 25$

M. S. Robertson, Cesàro partial sums of harmonic series expansions...... 829

John L. Selfridge and Ernst Gabor Straus, On the determination of numbers by their sums of a fixed order ........................ 847

Annette Sinclair, A general solution for a class of approximation

problems .................................

George Szekeres and Amnon Jakimovski, $(C, \infty)$ and $(H, \infty)$ methods of summation...................................... 867

Hale Trotter, Approximation of semi-groups of operators. ............. 887

L. E. Ward, A fixed point theorem for multi-valued functions ........... 921

Roy Edwin Wild, On the number of lattice points in $x^{t}+y^{t}=n^{t / 2} \ldots \ldots .929$ 